Check for updates

AUTHOR:

J. Francis Thackeray

AFFILIATION:

'Evolutionary Studies Institute, University of the Witwatersrand, Johannesburg. South Africa

CORRESPONDENCE TO: Francis Thackeray

EMAIL:

mrsples@global.co.za

HOW TO CITE:

Thackeray JF. Doubling the age and size of the universe at the IAU in Rome in 1952: Contributions by David Thackeray, Walter Baade and Harlow Shapley. S Afr J Sci. 2020;116(1/2), Art. \#6363, 2 pages. https://doi.org/10.17159/ sajs.2020/6363

\section{ARTICLE INCLUDES:}

$\square$ Peer review

$\square$ Supplementary material

\section{KEYWORDS:}

astronomy, history of science, Magellanic Clouds, Radcliffe Observatory, South Africa

PUBLISHED:

29 January 2020 (c) 2020. The Author(s). Published under a Creative Commons Attribution Licence.

\section{Doubling the age and size of the universe at the IAU in Rome in 1952: Contributions by David Thackeray, Walter Baade and Harlow Shapley}

In September 1952, the International Astronomical Union (IAU) met in Rome. The meeting was attended by 430 astronomers from 34 countries. ${ }^{1}$ The inaugural ceremony took place in the historic palace at the Campidoglio on the Capitoline hill, and sessions were held in the building of the National Research Council near the Cittá Universitaria. The Vatican Observatory at Castel Gondolfo was visited in the course of the conference, and the degates were addressed by Pope Pius XII.

Commission 28 was convened to discuss extra-galactic nebulae. It was attended by, among others, Georges Lemaitre (proposer of the concept of the Big Bang), Fred Hoyle (proponent of a 'steady state' universe) and Jan Oort (after whom the Oort Clouds are named). Without doubt, the most remarkable event at the meeting of this Commission was the recognition that the perceived age and size of the universe had to be doubled. Douglas ${ }^{1}$ described the session as 'intensely interesting'. Three astronomers were central in this connection (Figure 1): Walter Baade (a German, based at the Mount Wilson Observatory in California, USA); my late father, David Thackeray (trained in Cambridge in the UK, and subsequently based in Pretoria, South Africa); and Harlow Shapley (trained at Princeton and based at Harvard, Massachusetts, USA).

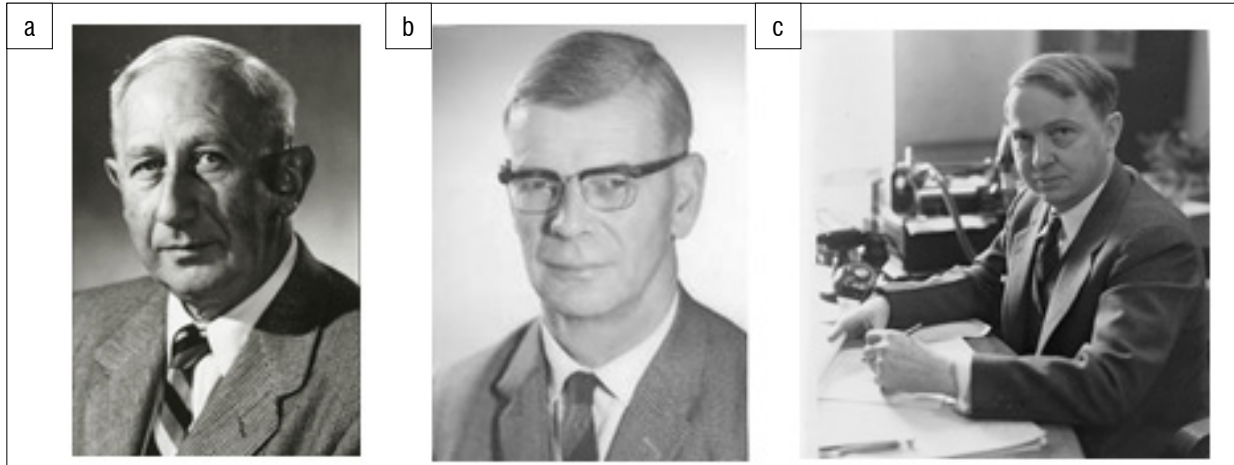

Sources: $\quad$ (a) Wikimedia, (b) Wikitree, (c) Scoopnest

Figure 1: (a) Walter Baade, (b) David Thackeray and (c) Harlow Shapley.

Prior to 1952, Shapley had been able to calibrate a period-luminosity relationship which could be used in efforts to determine distances on the basis of observations of stars of variable brightness, including Cepheids ('standard candles') visible in the northern hemisphere. ${ }^{2}$ For southern hemisphere observations, Shapley made use of the 24-inch Bruce refractor telescope at the Boyden Station on 'Harvard Koppie' near Bloemfontein in South Africa. Since 1931 (with Edwin Hubble), Baade had been using the 100-inch reflector telescope on Mount Wilson, and in particular attempted to determine the distance of the Andromeda Nebula (M31) using Cepheid variables. After 1948, Thackeray used the 74-inch reflector telescope that had just begun operations in Pretoria on 'Oxford Koppie' at the Radcliffe Observatory which, at that time, was the largest telescope in the southern hemisphere. (This telescope was moved to Sutherland in 1974 on account of light pollution in Pretoria).

Baade had written to Thackeray in 1949, encouraging him to look for RR Lyrae variable stars in the Magellanic Clouds. The two men had known each other in 1934 when a 24-year-old Thackeray was based at Mount Wilson, and just embarking on an astronomical career in the stimulating presence of Hubble.

Feast $^{3}$ and Glass ${ }^{4}$ refer to the following correspondence from Baade to Thackeray in 1949:

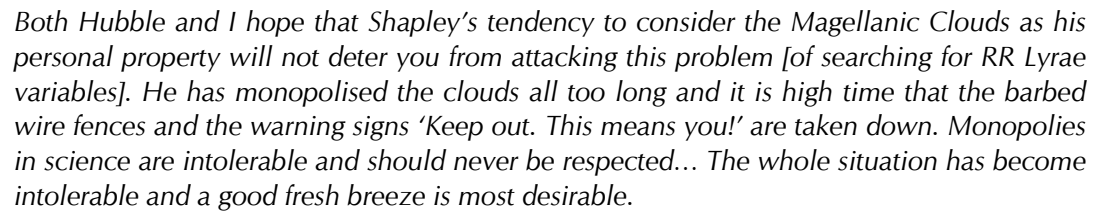

That 'fresh breeze' did indeed blow at the IAU in Rome in September 1952. Baade himself reported that RR Lyrae variables could not be detected in the Andromeda Nebula, even when using the 200-inch telescope at Mount Palomar in California. This by itself was a notable fact. Baade suggested that the distance of M31 might be greater than previously thought, with conjectural implications for an increase in the perceived size of the universe. Glass ${ }^{4}$ refers to an instantaneous sequel:

Immediately afterwards Thackeray made the dramatic and unexpected announcement that he had already found RR Lyraes in the Small Magellanic Clouds and could thus confirm Baade's suggestions. This had the consequence that the true Cepheids had to be more luminous than Shapley had thought, and that therefore he had underestimated their distances. One consequence was that the universe had to be twice as large as previously believed. 
In 1952, Shapley said that he could no longer be regarded as 'Mr Magellanic Clouds', in deference to David Thackeray and his Dutch colleague Adriaan Wesselink based at the Radcliffe Observatory. ${ }^{4}$ Subsequently, Shapley appears to have claimed credit for doubling the perceived size of the universe. At a meeting of the American Astronomical Society he presented the 'distance scale' based on variable stars in the Magellanic Clouds and the Andromeda Nebula. The sensational but unpublicised conclusions that had initially been announced quietly at the IAU in Rome in 1952 were presented (in Shapley's name) in The New York Times and elsewhere. Baade took umbrage and stated that it was 'simply shameless' for Shapley to claim credit; Bart Bok stated that he 'did not like it'; and 'a concerted effort was made by leading American astronomers to make sure that the true facts became known'4

Dubow ${ }^{5}$ has recently published an article on the history of astronomy in South Africa. He writes:

\begin{abstract}
Professor David Thackeray's astronomical research extended back to 1952, when he announced at the International Astronomical Union meeting in Rome that he and Wesselink had found evidence of RR Lyrae variables in the Magellanic Clouds. This dramatic discovery revealed that the universe was double the size of current estimates - thus resolving a puzzle whereby the earth seemed to be older than the universe itself. Much of the credit for the discovery went to internationally renowned astrophysicist Walter Baade, in part because Thackeray was overly diffident in publicising his findings. It may well be that the international world of astronomy [in 1952] was not yet prepared to accept that fundamental scientific findings could emanate from distant South Africa. Michael Feast, whose career as an astronomer in South Africa extends over half a century of active work, summarises the overall contribution of the Radcliffe Observatory thus: 'It enabled astronomers to begin to place the study of the southern hemisphere (with its exceptional globular clusters, the Magellanic Clouds, the Galactic Centre, etc.) on a par with that of the north.
\end{abstract}

In 1953 and 1954, Thackeray and Wesselink ${ }^{6,7}$ formally published the results of their combined research on RR Lyrae variables, notably in NGC 121 in the Small Magellanic Cloud (Figure 2) which had been studied by Henrietta Leavitt in 1912 in the context of period-luminosity relationships. NGC 121 is now estimated as being about 200000 light years away, and about 10 billion years old. Even older stars are known in the Large Magellanic Cloud.

In his Bruce Medal address in Pasadena in 1955, Baade reflects the excitement of the moment at the IAU meeting in Rome 8,9 , when Thackeray 'rose to announce data obtained at the Radcliffe Observatory'.

Trimble ${ }^{10}$ refers to the contribution by both Baade and Thackeray in reestimating the Hubble constant, relating to the expansion of the universe. Whereas Lemaitre and Hubble had initially suggested a $\mathrm{H}_{0}$ value of between 500 and $600 \mathrm{~km} / \mathrm{s} . M p c$ in about 1925, this was essentially halved in 1952 to $280 \pm 30 \mathrm{~km} / \mathrm{s}$.Mpc by Thackeray and Baade. ${ }^{10}$ Since then, its determination has been improved to circa $74 \mathrm{~km} / \mathrm{s} . \mathrm{Mpc}$.

Thackeray was very modest in his accomplishments, but he is known to have said in private that he considered his announcement in Rome to have been his greatest achievement in his entire career, when he contributed to the doubling of the perceived size and age of the universe on the basis of meticulous observations of RR Lyrae variables in the Magellanic Clouds, under challenging circumstances. In 1952 at the Radcliffe Observatory in Pretoria, the precise orientation of the telescope in the direction of a star was controlled visually through the eyepiece, and glass photographic plates were developed in a dark room, prior to the advent of computerisation and digitisation. The modernised telescope continues to be used, adjacent the Southern African Large Telescope (SALT) at Sutherland in the Karoo where
Thackeray was tragically killed in a road accident in 1978 at a time when he was still actively involved with research.

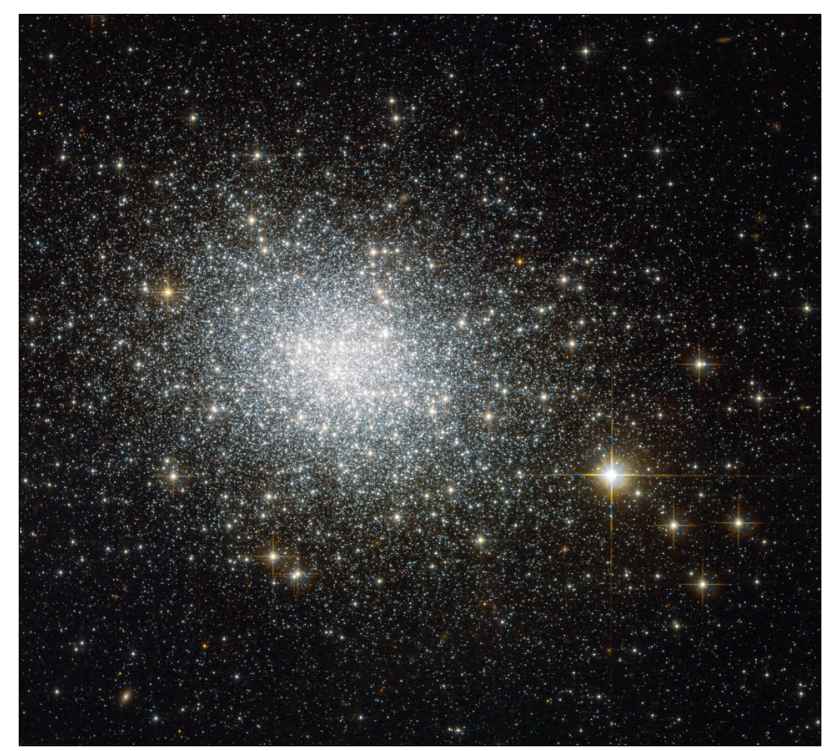

Image CC-BY: ESA/Hubble \& NASA, Stefano Campani, 2014

Figure 2: NGC 121, a globular cluster in the Small Magellanic Cloud discovered by John Herschel in South Africa in 1835. It is now recognised as being about 200000 light years away and about 10 billion years old. Thackeray and Wesselink ${ }^{6,7}$ used RR Lyrae variable stars in this globular cluster to double the size and age of the universe as perceived in 1952, when results obtained from the Radcliffe Observatory in Pretoria were announced at a conference of the International Astronomical Union in Rome, supplementing data obtained by Walter Baade and Edwin Hubble who studied the Andromeda Nebula (M31) at the Mount Wilson Observatory in California. Variable stars in NGC 121 had been studied by Henrietta Leavitt in 1912 to determine a periodluminosity relationship. Harlow Shapley was able to calibrate the relationship to estimate distances of stars of variable brightness.

\section{Acknowledgements}

I thank lan Glass and Robin Catchpole for their comments on an initial draft of the manuscript of this article which is intended as a note of historical interest.

\section{References}

1. Douglas AV. Eighth General Assembly of the International Astronomical Union. J Roy Astronom Soc Can. 1952;46(6):217-221.

2. Gingerich 0J. Harlow Shapley and Mount Wilson. Bull Am Acad Arts Sci. 1973;26(7):10-24. https://doi.org/10.2307/3822625

3. Feast MW. Stellar populations and the distance scale: The BaadeThackeray correspondence. J Hist Astron. 2002;31:29-36. https://doi org/10.1177/002182860003100102

4. Glass I. Revolutionaries of the cosmos: The astrophysicists. Oxford: Oxford University Press; 2006

5. Dubow S. 200 Years of astronomy in South Africa: From the Royal Observatory to the 'Big Bang' of the Square Kilometre Array. J South Afr Stud 2018;45(4):663-687. https://doi.org/10.1080/03057070.2018.1496700

6. Thackeray AD, Wesselink AJ. Distances of the Magellanic Clouds. Nature. 1953;171:693. https://doi.org/10.1038/171693a0

7. Thackeray AD, Wesselink AJ. Distances of the Magellanic Clouds (II) The Observatory. 1955;75:33-34.

8. 28-Commission des nebuleuses extragalactiques. Trans Int Astron Union 1954;8:397-399.

9. Baade W. The period-luminosity relation of the Cepheids. Pub Astron Soc Pacific. 1955;68:5-16. https://doi.org/10.1086/126870

10. Trimble V. HO: The incredible shrinking constant 1925-1975. Pub Astron Soc Pacific. 1996;108:1073-1082. https://doi.org/10.1086/133837 